\title{
Does MIDH Inhibitors is a Promising Therapy?
}

\author{
Nahla A M Hamed* \\ Professor of Hematology, Faculty of Medicine, Alexandria University, Egypt \\ Received: July 11, 2017; Published: July 14, 2017 \\ *Corresponding author: Nahla A M Hamed, Professor of Hematology, Faculty of Medicine, Alexandria University, Egypt
}

\begin{abstract}
IDH are key metabolic enzymes that play a role in mitochondrial oxidative phosphorylation, glutamine metabolism, lipogenesis, glucose sensing, and regulation of cellular redox status. Mutations in IDH1 and IDH2 proteins catalyze the reduction of $\alpha$-KG to 2-HG. The abnormal production of the oncometabolite 2-HG promote tumorigenesis. Depending on the associated genomic aberrations and the cellular context, the oncogenic potential of $I D H 1 / 2$ mutations ranges from an initiating event - promoting transformation - to a secondary oncogenic event conferring selective advantage to cancer cells.
\end{abstract}

Abbreviation: IDH1 and IDH2: Isocitrate Dehydrogenase 1 and 2; $\alpha$-KG:- $\alpha$ ketoglutarate; 2-HG: 2-hydroxyglutarate; TET: Ten Eleven Translocation; mIDH: Mutant IDH; HIF: Hypoxia Induced Factor; PARP: Polyadenosine 5' Diphosphate Ribose; HR: Homologous Recombination

\section{Introduction}

IDH are family of enzymes that comprises three proteins, IDH1, IDH2 and IDH3. IDH1 is located in the cytoplasm while IDH2 and IDH3 are located in the mitochondria [1,2]. The IDHs are tricycle carboxylic acid cycle enzymes which convert isocitrate to $\alpha$-KG via oxidative decarboxylation, producing NADPH (IDH1 and 2) and NADH (IDH3) [3]. IDH proteins are involved in diverse cellular processes including adaptation to hypoxia, histone demethylation and DNA modification [4]. Mutations have only been identified in IDH1 and IDH2 gene [3]. The mutated IDH1 and IDH2 proteins have a gain of function, neomorphic activity, catalyzing the reduction of $\alpha$-KG to 2-HG [5]. The abnormal production of the oncometabolite 2-HG promote tumorigenesis [5]. IDH1/2 mutations define distinct subsets of cancers, including low-grade gliomas, secondary glioblastomas, chondrosarcomas, intrahepatic cholangiocarcinomas, and hematologic malignancies [6].

MIDH1/2 is among the most common mutations in AML. IDH1 mutations are less common than IDH2 mutations in AML [7]. Mutations in genes encoding IDH1 and IDH2 gene isoforms occur in $6-16 \%$ and $8-19 \%$ of AML cases, respectively [3] ( $20 \%$ of patients have combined mutation) [7]. The frequency of mutations in these genes increases with age at diagnosis [3]. mIDH are less frequent in MDS $(\sim 5 \%)$ and myeloproliferative neoplasms. The frequency increases to $\sim 20 \%$ in myeloproliferative neoplasm at leukemic transformation [7].

\section{Isocitrate Dehydrogenase 1 and 2 in AML:}

In myeloid malignancies, IDH1 mutations most often involve a cysteine (R132C) or histidine (R132H) substitution for arginine at R132. In IDH2 mutations, arginine is most often replaced by glutamine at residue 140 (R140Q) and by lysine at residue 172 (R172K). IDH2-R140 mutations are more common than IDH2-R172 mutations, representing $\sim 80 \%$ of IDH2 mutations in AML. IDH2-R172 mutations may be more frequent in older patients. Less frequently, other amino-acid substitutions are involved (for example, IDH2-R172W) [7]. These mutations must occur in a heterozygous form to be able to generate mutant heterodimers. The wild-type enzyme produces $\alpha-\mathrm{KG}$, and the mutant subunit monomer swiftly reduces $\alpha-K G$ to its functional antagonist, 2-HG [3].

mIDH1 and mIDH2 have different enzymatic activities. Cytoplasm mIDH1 generates less 2-HG than mitochondrial mIDH2 enzyme. This may be due to differences in amounts of $\alpha-K G$ substrate, which is found in greater abundance in the mitochondrion than in the cytoplasm [7]. 2HG oncometabolite accumulation promotes tumorigenesis through inhibition of a cancer-associated transcription factor such as HIF [1]. Moreover, $2-\mathrm{HG}$ is structurally similar to $\alpha-\mathrm{KG}$ and competitively inhibits $\alpha$-KG-dependent enzymes, including [7] histone demethylases and methyl cytosine dioxygenases of the TET family that regulate cellular epigenetic status [8]. TET2 protein is involved in DNA demethylation. Histone demethylases regulate chromatin status, enabling activation or inhibition of gene transcription [7]. This epigenetic dysregulation produces global histone- and DNA hypermethylation [1] characterized by an increased methylation of promoter sites of target genes [4], altered gene expression leading to differentiation arrest of hematopoietic progenitors [7] and a maturation block [3] which potentially lead to leukemogenesis [1]. mIDH may also exist in preleukemic stem cells [7]. 
IDH1 and IDH2 mutations in newly diagnosed AML patients have a strong association with AML-M2 subtype and NPM1 mutation [1]. IDH1 mutation was significantly associated with NPM1 mutation, in $12.5 \%$ to $67 \%$ as compared to the wild-type IDH1 cases. IDH2 mutations were significantly associated with NPM1 mutations in $60 \%$ of the cases [1]. They are almost always mutually exclusive with TET2 mutations and with mutations in the Wilms' tumor 1 gene [7]. IDH1 and IDH2 mutations are enriched in AML with normal cytogenetic (CN-AML), but may be present along with other cytogenetic abnormalities in $30 \%$ of the cases [3]. They are associated with cytogenetically intermediate-risk disease, and occur often with trisomy 8 [7]. No significant correlation with other mutations such as FLT3, RAS, and AML1 could be demonstrated [1]. The prognostic impact of IDH1/2 mutations in AML has been conflicting. Early data suggested adverse impact of IDH mutations in AML while recent data suggest that site of mutation (IDH1-R132, IDH2-R140, IDH2-R172) and co-occurrence with other mutations influences outcome. Co-occurrence of IDH2R140 with DNMT3a mutation confers worse prognosis while IDH2R172 mutation, though uncommon, is associated with favorable outcome. While survival is better in IDH1/2-mutated AML with concurrent NPM1 mutations, it compares less favorably against cytogenetically normal AML with NPM1 mutation alone [3]. Evidence was not considered sufficient to definitively categorize aberrations in IDH ( 1 or 2 ) into either the intermediate or adverse group, but may be useful in recommending patients for specific therapies [9].

\section{Inhibitors of Mutant IDH}

These therapies when used as single agents have shown promise for inducing responses in some mIDH patients as a first line treatment or in relapsed or refractory AML [4]. Preliminary results from phase 1 clinical trials with IDH inhibitors in patients with advanced hematologic malignancies have demonstrated an objective response rate ranging from $31 \%$ to $40 \%$ with durable response ( $>1$ year) observed [5]. Unlike other chemotherapeutics, these drugs are not cytotoxic and thus expected to be associated with lower rates of cytopenic complications [3]. Use of mIDH inhibitors in combination with drugs with non-overlapping mechanism of action is especially promising, as such regimens may address the clonal heterogeneity and the multifactorial pathogenetic process involved in mIDH myeloid malignancies [4].

Current targeted inhibitors of IDH1 (AG120, IDH-305, FT-2102), IDH2 (AG221), and a pan- IDH1/2 (AG881) selectively inhibit mIDH protein and induce cell differentiation in in vivo and in vitro models [5]. Enasidenib (AG-221) has been accepted by U.S. FDA in 2017 for treatment of patients with relapsed or refractory AML with IDH2 mutation. AGI-6780, a selective sulfonamide inhibitor of the mutant IDH2 enzyme, lowered 2HG levels and induced differentiation of TF-1 erythroleukemia cells and primary human AML cells harboring the IDH2R140Q mutation [8]. BAY 1436032, a novel pan-mutant IHD1 inhibitor that inhibits $2 \mathrm{HG}$ production and colony growth and induces myeloid differentiation of AML cells carrying all major types of IDH1 mutant AML (IDH1R132H,
IDH1R132C, IDH1R132G, IDH1R132L, and IDH1R132S). In addition, the compound attenuates histones hypermethylation in patient derived xenograft IDH1 mutant mouse models [10].

\section{Future Therapy}

Elevated 2-HG levels can lower mitochondrial threshold for triggering apoptosis through BCL-2 inhibition such as venetoclax. 2-HG production sensitizes mutant cells to ATRA-induced differentiation. 2-HG causes defect in HR-mediated DNA doublestrand break repairs. This renders mutant cells sensitive to PARP inhibition [3]. IDH mutations induce cancer cells dependency for glutamine as the main source of 2-HG. This can be tested by a potent glutaminase inhibitor, CB-839 (NCT02071927) [3]. Inhibitors of an epigenetic 'reader' protein, Brd4 can produce differentiation and cell death of mIDH $1 / 2$ AML cell. Hypomethylating agents may be effective against mIDH1/2 global hypermethylation [3]. IDH1/2 mutants are ideal tumor-specific neoantigens due to their uniform occurrence at specific codons and ubiquitous expression throughout all tumor cells. These suggest that mutant IDH1targeted immunotherapies can elicit potent antitumor immune responses [6].

\section{Conclusion}

The therapeutic potential of mIDH inhibitors can pave the way for treatment individualization to improve outcomes.

\section{References}

1.Chotirat S, Thongnoppakhun W, Promsuwicha O, Boonthimat C, Auewarakul CU (2012) Molecular alterations of isocitrate dehydrogenase 1 and 2 (IDH1 and IDH2) metabolic genes and additional genetic mutations in newly diagnosed acute myeloid leukemia patients. J Hematol Oncol 5: 5-14

2.Stein EM (2015) IDH2 inhibition in AML: finally progress? Pract Res clin Haematol 28(2-3): 112-115.

3.Boddu P, Borthakur G (2017) Therapeutic targeting of isocitrate dehydrogenase mutant AML. Expert Opin Investig Drugs 26(5): 525-529.

4.Medieros BC, Fathi AT, DiNardo CD, Pollyea DA, Chan SM, et al. (2017) Isocitrate dehydrogenase mutations in myeloid malignancies. Leukemia 31(2): 272-281.

5.Fujii T, Kawaja MR, DiNardo CD, Atkins JT, Janku F. (2016) Targeting isocitrate dehydrogenase in cancer. Discov Med 21(117): 373-380.

6.Mondesir J, Willekens C, Touat M, deBotton S. (2016) IDH1 and IDH2 mutations as novel therapeutic targets: current perspectives. J Blood Med 7: 171-180.

7.Medeiros BC, Fathi AT, DiNardo CD, Pollyea DA, Chan SM, et al. (2017) Isocitrate dehydrogenase mutations in myeloid malignancies. Leukemia 31(2): 272-281.

8.Yen K, Travins J, Wang F, David MD, Artin E, et al. (2017) AG-221, a first-inclass therapy targeting acute myeloid leukemia harboring oncogenic IDH2 mutations. Cancer Discov 7(5); 478-493.

9.Tefferi A, Estey EH (2016) Acute myeloid leukemia: 2016 Update on riskstratification and management. Am J Hematol 91(8): 824-846.

10. Chaturvedi A, Herbst A, Pusch S, Klett L, Goparaiu R, et al. (2017) Panmutant-IDH1inhibitor BAY1430632 is highly effective against human IDH1 mutant acute myeloid leukemia in vivo. Leukemia. 


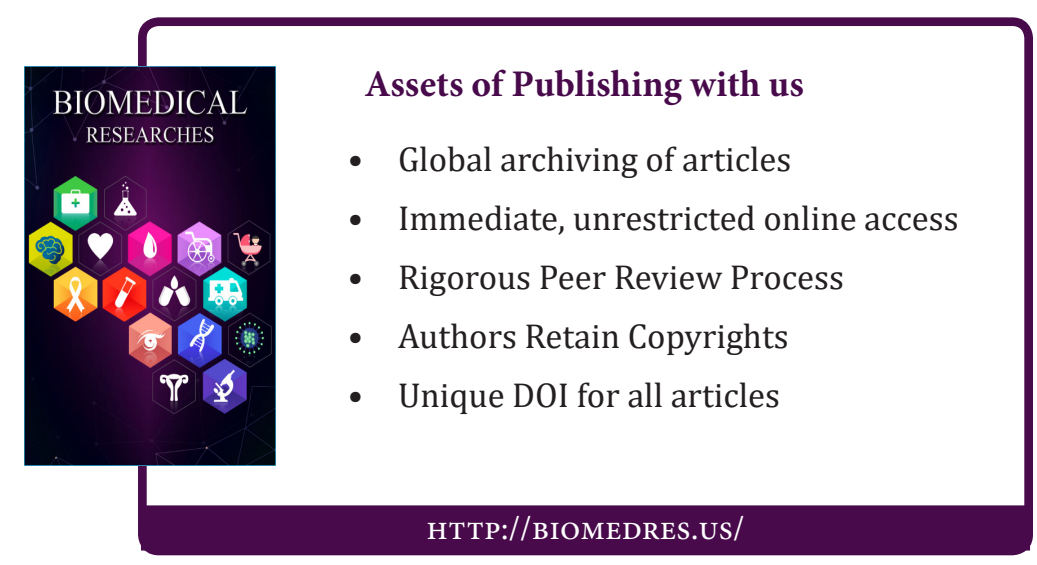

